\title{
Lista das espécies de Bibionomorpha (Diptera) do Estado do Mato Grosso do Sul, Brasil
}

\author{
Sarah Siqueira Oliveira', Rafaela Lopes Falaschi², \\ Maria Virginia Urso-Guimarães ${ }^{3} \&$ Dalton de Souza Amorim ${ }^{4}$
}

\footnotetext{
1. Universidade Federal de Goiás, Campus II. Instituto de Ciências Biológicas, Departamento de Ecologia, prédio ICB1. Av.Esperança, s/n, Campus Samambaia, 74.690-900, Goiânia, Goiás, Brasil. (oliveira.sarahcv@gmail.com)

2. Universidade de São Paulo, Museu de Zoologia, Laboratório de Diptera, Av. Nazaré, 481, 04263-000 São Paulo, SP, Brasil.

3. Universidade Federal de São Carlos, Campus Sorocaba, Centro de Ciências Humanas e Biológicas, Departamento de Biologia, Rod. João Leme dos Santos, SP-264 Km 110, Sorocaba, SP, Brasil.

4. Universidade de São Paulo, Faculdade de Filosofia, Ciências e Letras de Ribeirão Preto, Departamento de Biologia, Av. Bandeirantes, 3900, 14040-901

Ribeirão Preto, SP, Brasil.
}

Recebido 29 novembro 2016

Aceito 6 fevereiro 2017

DOI: $10.1590 / 1678-4766 e 2017127$

ABSTRACT. Checklist of the Bibionomorpha (Diptera) of the State of Mato Grosso do Sul, Brazil. We provide in this paper a checklist of Bibionomorpha in the state of Mato Grosso do Sul, Brazil. Only the families Anisopodidae, Sciaridae, Keroplatidae, and Mycetophilidae have been recorded from this state so far: one genus and one species of Anisopodidae; three genera and nine species of Sciaridae; five genera and six species of Keroplatidae; and eight genera and 14 species of Mycetophilidae. A list of other species of Bibionomorpha families known from the Central-Western region of Brazil supposed to occur in the state of Mato Grosso do Sul is provided as well. This is a good example of the distance between real diversity of an important group as Diptera in a large area as the state of Mato Grosso do Sul, and the actual knowledge of the group.

KEYWORDS. Diversity, taxonomy, distribution, Central-Western region, Biota-MS Program.

RESUMO. Neste trabalho, é fornecida uma lista de espécies de Bibionomorpha conhecidas para o estado do Mato Grosso do Sul, Brasil. Das famílias dessa subordem de Diptera, apenas Anisopodidae, Sciaridae, Keroplatidae e Mycetophilidae foram registradas até o momento para essa região. Anisopodidae é representada por um gênero e uma espécie, Sciaridae por três gêneros e nove espécies, Keroplatidae por cinco gêneros e seis espécies, enquanto que Mycetophilidae é conhecida por oito gêneros e 14 espécies. São elencadas possíveis espécies das famílias de Bibionomorpha já conhecidas da região Centro-Oeste do Brasil cuja presença é esperada no Mato Grosso do Sul. Este levantamento é bastante indicativo da enorme diferença existente entre o conhecimento efetivo da biodiversidade de um grupo importante, como Diptera, em uma área grande, como o Mato Grosso do Sul, e a diversidade real do grupo em uma região do Brasil.

PALAVRAS-CHAVE. Diversidade, taxonomia, distribuição, Região Centro-Oeste, Programa Biota-MS.

A classificação dos grupos basais de Diptera foi recentemente revista (АMORIM \& YeATES, 2006). O táxon "Nematocera", sabidamente parafilético em relação a Brachycera, foi formalmente eliminado, suas infraordens passando a ser reconhecidas como subordens, entre elas, Bibionomorpha. A subordem Bibionomorpha é um grupo composto por Pachyneuridae, Bibionidae, Cecidomyiidae, Sciaridae, Rangomaramidae, Ditomyiidae, Bolitophilidae, Diadocidiidae, Keroplatidae, Lygistorrhinidae e Mycetophilidae (Wood \& Borkent, 1989; Matile, 1990; ChANDLER, 2002). A inclusão de Anisopodidae no grupo é motivo de alguma divergência na literatura. Essa família foi tratada como parte de Bibionomorpha (p. ex., HeNNIG, 1973; AMORIM, 1993; AMORIM \& Tozoni, 1994), como um clado independente - Anisopodomorpha - por TUOMIKOSKI (1961), como parte de Psychodomorpha (WoOd \& BORKENT, 1989) e como grupo-irmão de Brachycera (WoODLEY, 1989; Oosterbroek \& Courtney, 1995). Trabalhos recentes com base em dados morfológicos (AMORIM \& RINDAL, 2007) e moleculares (BERTONE et al., 2008; WIEGMANN et al., 2011) apontam para a inclusão de Anisopodidae em Bibionomorpha. Por outro lado, Scatopsidae e Canthyloscelidae, aceitos como parte de Bibionomorpha por HeNNIG (1973), foram indicados, com base em caracteres de imaturos, como pertencentes a Psychodomorpha por WOOD \& BORKENT (1989), posição depois aceita por AMORIM (1994), embora filogenias moleculares recentes voltem a apontar essas duas famílias como parte de Bibionomorpha (BERTONE et al., 2008; WIEGMANN et al., 2011), posição que não é adotada aqui.

Outra questão é a inclusão do gênero Hesperinus Walker como família independente ou como uma subfamília basal dentro de Bibionidae (Fitzgerald, 2009). Pinto \& AMorim (2000) fizeram uma análise filogenética de Bibionidae, apontando o grupo como irmão do restante da família, análise replicada por FitzGERALD (2004). PAPP (2010) descreveu novas espécies do gênero como membro 
de uma família à parte, mas sem uma análise filogenética mais abrangente. Neste trabalho, segue-se a classificação de PinTo \& AMORIM (2000).

Assim, Bibionomorpha é aceita aqui como sendo composta por 12 famílias recentes. Pachyneuridae e Bolitophilidae não possuem representantes neotropicais. Um amplo estudo das relações filogenéticas de Bibionomorpha foi realizado por AMORIM \& RINDAL (2007) será utilizado aqui para a classificação do grupo. Informações sobre características diagnósticas, chaves de identificação e biologia de cada família podem ser encontradas em BRown et al. (2009). Alguns trabalhos apontam que o número de espécies conhecidas para Diptera em algumas famílias pode ser de 10\% da diversidade real desses grupos nas regiões Neotropical, Oriental e Australiana (BRown, 2005; EvenHUIs, 2006), de modo geral, ou ao Brasil e ao Mato Grosso do Sul, em particular. De fato, uma estimativa ainda inicial mostra quase uma média de $30 \%$ de espécies novas de Diptera em florestas semidecíduas em relação ao número de espécies conhecidas, que para algumas famílias é de 100\% (AMORim et al., 2010).

Anisopodidae. Consiste de sete gêneros com 120 espécies descritas, amplamente distribuídas geograficamente no mundo (HANCOCK \& AMORIM, 2009). Cinco gêneros e 65 espécies são conhecidos para a Região Neotropical (AMORIM et al., 2002; HANCOCK \& AMORIM, 2009). AMORIM \& Tozoni (1994) aceitaram três táxons ao nível de família em Anisopodoidea - Olbiogastridae, Anisopodidae e Mycetobiidae -, mas essa estrutura tem sido pouco aceita, de maneira que no presente trabalho optou-se por manter Anisopodidae como família, seus três grupos principais como subfamílias. No Brasil, há registro de três gêneros (Carreraia Corrêa, Sylvicola Harris e Olbiogaster Osten Sacken), com cerca de 21 espécies descritas (PAPAVERO, 1967; TozonI, 1993; HANCOCK \& AMORIM, 2009).

Bibionidae. Atualmente conta no mundo com cerca de 700 espécies descritas para oito gêneros (FITZGERALD, 2009). Todos os gêneros possuem representantes neotropicais, com um total de 169 espécies (AMOrim et al., 2002). Há registros de sete gêneros e 40 espécies para o Brasil (Hardy, 1966; FitzGerald, 1997). Atualmente o grupo encontra-se organizado em quatro subfamílias, Hesperininae, Penthetriinae, Pleciinae e Bibioninae (Pinto \& Amorim, 2000; FitzGerald, 2009; Papp, 2010), sendo que as três primeiras são monotípicas. No Mato Grosso do Sul, esperase encontrar os gêneros Dilophus Meigen, Bibio Linnaeus, Bibionellus Edwards e Enicoscolus Hardy.

Cecidomyiidae. É a família mais numerosa e diversa da subordem, com 6.203 espécies descritas para o mundo. Aceitam-se seis subfamílias em Cecidomyiidae: cinco subfamílias basais com hábito micófago, Catotrichinae, Lestremiinae, Micromyinae, Winnertziinae e Porricondylinae, e Cecidomyiinae, a subfamília mais apical que compreende todas as espécies com hábito galhador e inclui cerca de $80 \%$ das espécies da família (GAGNÉ \& JASCHHOF, 2014). Para a Região Neotropical, são conhecidos cerca de 605 espécies de 173 gêneros, dos quais 87 espécies de 80 gêneros relatados para o Brasil (GAGNÉ \& JASCHHOF, 2014). Não há espécies apontadas no catálogo do grupo para o Mato Grosso do Sul. Sciaridae. Atualmente há cerca de 2.400 espécies conhecidas em cerca de 90 gêneros no mundo (VILKamaA \& HipPA, 2004; Mohrig \& Menzel, 2009). Para o Brasil, há 201 espécies descritas, distribuídas em 29 gêneros (AMORIM, 1992). A taxonomia genérica da família tem mudado bastante nos últimos anos devido especialmente à sinonímia de gêneros. Estudos iniciais da diversidade de alguns gêneros apontam para uma enorme diversidade ainda a ser descrita, de até $90 \%$ em alguns gêneros, especialmente na região Neotropical (VILKAMAA \& HIPPA, 2004; HIPPA \& VILKAMAA, 2005). HipPa \& VilKamaA (2005) propuseram algumas subfamílias, mas a falta de dados não permite que os gêneros conhecidos para a Região Neotropical, por exemplo, sejam incluídos nesse sistema (Mohrig \& Menzel, 2009).

Rangomaramidae. A família foi estabelecida por JASCHHOF \& DidHAM (2002) para cinco espécies de Rangomarama Jaschhof \& Didham, da Nova Zelândia. O conceito da família foi revisto por AMORIM \& RINDAL (2007), a partir de um estudo filogenético de Mycetophiliformia, adicionando gêneros cuja posição até então era altamente controversa. Rangomaramidae passou, então, a abranger no mundo 11 gêneros e cerca de 40 espécies em quatro subfamílias - Rangomaraminae, Heterotrichinae, Chiletrichinae e Ohakuneinae. Seis gêneros e 20 espécies são conhecidos para a Região Neotropical, sendo Chiletricha marginata (Edwards) e Colonomyia brasiliana Amorim \& Rindal conhecidos do sudeste do Brasil (AMORIM \& FALASCHI, 2012). Espera-se que ao menos Colonomyia Colless seja registrado em áreas mais altas do Mato Grosso do Sul.

Ditomyiidae. É uma pequena família com 15 gêneros e cerca de 107 espécies, conhecida em todas as regiões biogeográficas exceto a Afrotropical. Há 32 espécies descritas para a Região Neotropical, distribuídas em nove gêneros (MunRoE, 1974; PAPAVERO, 1978a). No Brasil, ocorrem quatro dos gêneros descritos, com cerca de 14 espécies (FALASCHI, 2008; FAlaschi \& Amorim, 2009). O número de espécies novas no grupo em áreas de florestas tropicais, pertencentes a Rhipidita Edwards, Calliceratomyia Lane e Australosymmerus (Melosymmerus) Munroe, é particularmente alta, de forma que se espera encontrar registros desses gêneros em áreas com características similares no Mato Grosso do Sul.

Diadocidiidae. Diadocidiidae é uma das menores famílias de Bibionomorpha, com um gênero e 24 espécies (Bechev \& Chandler, 2011). A família é conhecida das regiões Neártica, Paleártica, Oriental, Australiana/Oceania e Neotropical. Para a Região Neotropical, PAPAVERo (1977a) mencionou Diadocidia nigripalpis Edwards para o sul do Brasil (Santa Catarina) e uma espécie ainda não descrita para o Chile. JASCHHOF \& JASCHHOF (2007) redescreveram D. nigripalpis e ampliaram sua distribuição, que alcança a Costa Rica, indicando a espécie como amplamente distribuída na Região Neotropical. Diadocidia Ruthe certamente está presente no Mato Grosso do Sul. 
Keroplatidae. Atualmente compreende cerca de 950 espécies no mundo, distribuídas em 92 gêneros (PAPAVERO, 1978b; Matile, 1990; Evenhuis, 1994; 2006; PaPP, 2008; ŠEvČíK, 2009; ŠEvČÍK \& PAPP, 2009; CoHer, 2011; PAPE et al., 2011), incluídos em Arachnocampinae, Macrocerinae e Keroplatinae (Matile, 1981). PApp \& Ševčík (2005) propuseram Sciarokeroplatinae para uma única espécie, Sciarokeroplatus pileatus Papp \& Sevčik, da região Oriental, mas o status de subfamília para esse gênero não tem sido aceito na literatura. Na Região Neotropical, atualmente encontram-se 32 gêneros e mais de 200 espécies, das quais 40 são conhecidos do Brasil, especialmente das regiões Sul e Sudeste (Evenhuis, 2006; Falaschi, 2012).

Lygistorrhinidae. A fauna vivente da família divide-se em oito gêneros e 32 espécies para o mundo, em especial em áreas tropicais e subtropicais (HIPPA et al., 2005; HUERTA \& IbaÑEZ-Bernal, 2008). Na Região Neotropical, (PAPAVERo, 1977b; 2002; Oliveira \& AMORIM, 2012a), há apenas um gênero e nove espécies, das quais cinco são conhecidas para o Brasil (PAPAVERo, 1977b; 2002; OliveIra \& AMORIM, 2012a). A presença de Lygistorrhina (Probolaeus) Williston no Mato Grosso do Sul é praticamente certa.

Mycetophilidae. É a segunda família mais numerosa e diversificada de Bibionomorpha com 180 gêneros viventes e cerca de 4.500 espécies descritas de todas as regiões biogeográficas (PAPE et al., 2011). Apesar de não haver um consenso sobre as relações filogenéticas dentro de Mycetophilidae, o monofiletismo do grupo é corroborado com dados morfológicos (Sølı, 1997; Tozoni, 1998) e moleculares (RINDAL et al., 2009). Nas classificações mais recentes, os micetofilídeos encontram-se divididos em sete subfamílias: Sciophilinae, Gnoristinae, Mycomyiinae, Leiinae, Manotinae, Allactoneurinae e Mycetophilinae (TuOMiKoski, 1966; HeNnig, 1973; VäISÄNEN, 1984; Matile, 1989; Rindal et al., 2009), das quais apenas Allactoneurinae não apresenta representantes neotropicais. Em alguns casos, um grupo erigido por MatiLE (1971) como uma tribo de Gnoristinae - Metanepsiinae - é tratado como subfamília independente (VÄISÄNEN, 1984; ŠEVČíK \& HIPPA, 2010), mas isso certamente deixaria os Gnoristinae parafiléticos. Mycetophilidae na Região Neotropical inclui aproximadamente 54 gêneros e 1.145 espécies (Oliverra \& AMORIm, 2014). No Brasil, são conhecidos 39 gêneros e aproximadamente 360 espécies, a maioria das regiões Sul e Sudeste do país (PapaVero, 1978c; Oliveira \& AMOrim, 2014). Pelo menos metade dos gêneros de Mycetophilidae devem ter registros no Mato Grosso do Sul.

Fontes de Informação. Foi realizado um levantamento da bibliografia taxonômica de todas as famílias de Bibionomorpha, visando levantar as espécies conhecidas para o estado do Mato Grosso do Sul. Utilizaram-se os registros nos catálogos (mundiais e/ou neotropicais) de cada família, além de trabalhos complementares publicados sobre os grupos que mencionassem a distribuição de espécies na região Neotropical. Os trabalhos elencados para as famílias das quais há registros para o Mato Grosso do Sul são: Anisopodidae
(PAPAVERo, 1967; Tozoni, 1993 e Amorim \& Tozoni, 1994); Sciaridae (Amorim, 1992; Menzel et al., 2003 e OzdiKmen \& BASAR, 2011); Keroplatidae (PAPAVERO, 1978b; EvenhuIS, 2006; FALASCHI, 2012 e FalasCHI \& Amorim, dados inéditos); Mycetophilidae (PAPAVERO, 1978c; AMORIM et al., 2008a,b; Amorim \& Oliveira, 2008; Oliveira, 2009; Oliveira \& AMORIM, 2010a,b, 2011, 2012b, 2014).

Para aquelas famílias ainda sem registros de espécies para o Mato Grosso do Sul destacam-se os catálogos mundiais de Cecidomyiiidae (GAGNÉ \& JASCHHOF, 2014) e Diadocidiidae (Bechev \& ChANDler, 2011) e os catálogos de Bibionidae (HARDY, 1966), Rangomaramidae (AMORIM \& Falaschi, 2012), Ditomyiidae (PapaVero, 1978a), Diadocidiidae (PAPAVERO, 1977a) e Lygistorrhinidae (Papavero, 1977b; 2002; Oliveira \& AMOrim, 2012a). Trabalhos adicionais relevantes incluem FITZGERALD (1997), AMORIM \& Rindal (2007) e FALASCHI (2008).

Lista das espécies de Bibionomorpha do Mato Grosso do Sul. As espécies de Bibionomorpha conhecidas para o estado do Mato Grosso do Sul são listadas abaixo, cada uma acompanhada da localidade-tipo e da distribuição geográfica. Em alguns casos, apenas se reproduz a informação sobre a ocorrência no estado de espécies com distribuição mais ampla, embora seja possível que a conspecificidade seja questionável. O motivo de manter-se o registro aqui é que, ainda que a espécie identificada de material do Mato Grosso do Sul possa ser outra, deverá ser espécie do mesmo gênero, de modo que a lista estaria mais próxima do conhecimento da diversidade dos gêneros no estado do que com sua omissão.

\section{ANISOPODIDAE Olbiogaster Osten-Sacken, 1886}

midas Tozoni, 1993. Localidade-tipo: Brasil, Mato Grosso do Sul, Dourados. Distribuição: Brasil (Mato Grosso do Sul).

\section{SCIARIDAE Bradysia Winnertz, 1867}

forattinii (Lane, 1959). Localidade-tipo: Brasil, Mato Grosso do Sul, Bataguassu (Amaurilândia). Distribuição: Brasil (Mato Grosso do Sul).

matogrossensis (Lane, 1959). Localidade-tipo: Mato Grosso do Sul, Bataguassu (Quiteriazinha). Distribuição: Brasil (Mato Grosso do Sul).

meinacusi (Lane, 1959). Localidade-tipo: Mato Grosso do Sul, Bataguassu (Rio Três Barras). Distribuição: Brasil (Mato Grosso do Sul, Goiás, Rio de Janeiro, São Paulo). Ref.: AMORIM (1992).

\section{Eugnoriste Coquillett, 1896}

pernitens Edwards, 1941. Localidade-tipo: Brasil, Mato Grosso do Sul, Salobra. Distribuição: Brasil (Mato Grosso do Sul). 


\section{Pseudosciara Schiner, 1868}

grisapex Edwards, 1941. Localidade-tipo: Brasil, Mato Grosso do Sul, Salobra. Distribuição: Brasil (Mato Grosso do Sul).

coxalba Edwards, 1941. Localidade-tipo: Brasil, Mato Grosso do Sul, Salobra. Distribuição: Brasil (Mato Grosso do Sul).

hirtella Schiner, 1868. Localidade-tipo: "Colômbia". Distribuição: Colômbia, Brasil (Mato Grosso do Sul). Ref.: EDWARDS (1941).

thoracica (Lengersdorf, 1930). Localidade-tipo: Costa Rica, Farma La Caja (W San José). Distribuição: Costa Rica, Brasil (Mato Grosso do Sul). Ref.: EdwARDs (1941).

trifasciata (Coquillett, 1904). Localidade-tipo: Nicarágua, San Marcos. Distribuição: Nicarágua, Brasil (Mato Grosso do Sul). Ref.: EDWARDs (1941).

\section{KEROPLATIDAE \\ Macrocerinae Macrocera Meigen, 1800}

shannoni Lane, 1947. Localidade-tipo: Brasil, Mato Grosso do Sul, Maracaju. Distribuição: Brasil, Mato Grosso do Sul.

\section{Keroplatinae Apyrtula Edwards, 1940}

abbrevinervis (Edwards, 1941). Localidade-tipo: Brasil, Mato Grosso do Sul, Salobra. Distribuição: Brasil, Mato Grosso do Sul.

\section{Neoceroplatus Edwards, 1941}

minimax (Edwards, 1934). Localidade-tipo: Costa Rica. Distribuição: Argentina, Brasil (Mato Grosso do Sul, Goiás, São Paulo, Santa Catarina), Costa Rica, Panamá, Peru. Ref.: EdwARds (1941); LANE (1948); EvenHUIS (2006).

punctipes Matile, 1990. Localidade-tipo: Brasil, Mato Grosso do Sul, Salobra. Distribuição: Brasil (Mato Grosso do Sul).

\section{Placoceratias Enderlein, 1910}

bimaculipennis Enderlein, 1910. Localidade-tipo: Brasil, Mato Grosso do Sul, Maracaju. Distribuição: Brasil (Mato Grosso do Sul, Santa Catarina). Ref.: Lane (1948); EvenHuis (2006).

\section{Plautyra Edwards, 1941}

salobrensis (Edwards, 1941). Localidade-tipo: Brasil, Mato Grosso do Sul, Salobra. Distribuição: Brasil (Mato Grosso do Sul), Panamá, Trinidad. Ref.: Evenhuis (2006). Ref.: Amorim \& Oliveira (2008, 2013).

\section{MYCETOPHILIDAE \\ Sciophilinae \\ Cluzobra Edwards, 1940}

lanei Edwards, 1941. Localidade-tipo: Brasil, Mato Grosso do Sul, Salobra. Distribuição: Brasil (Mato Grosso do Sul e São Paulo). Ref.: Amorim \& OliveIRA (2008, 2013).

\section{Gnoristinae \\ Dziedzickia Johannsen, 1909}

cryptura Edwards, 1941. Localidade-tipo: Brasil, Mato Grosso do Sul, Salobra. Distribuição: Brasil (Pernambuco, Alagoas, Sergipe, Mato Grosso do Sul, Minas Gerais, Espírito Santo, Rio de Janeiro, São Paulo, Santa Catarina). Ref.: Oliveira (2009); Amorim \& Oliveira (2013).

medea Lane, 1954. Localidade-tipo: Brasil, São Paulo, São

Paulo (Cantareira). Distribuição: Argentina (Misiones), Brasil (Paraíba, Pernambuco, Mato Grosso do Sul, Minas Gerais, Rio de Janeiro, São Paulo, Santa Catarina). Ref.: OLIVEIRA, 2009.

\section{Mycomyiinae Mycomya Rondani, 1856}

clavata (Lynch Arribálzaga, 1892). Localidade-tipo: Argentina, Salta, Urundel. Distribuição: Argentina (Salta), Brasil (Mato Grosso do Sul). Ref.: EdwArds (1941). OLIVEIRA \& AMORIM (2014) erroneamente incluíram na distribuição da espécie Mycomya borinquensis Coher o estado de Mato Grosso do Sul, ao invés de Mato Grosso como previamente mencionado em PAPAVERO (1978c).

\section{Neoempheria Osten-Sacken, 1878}

pereirai Edwards, 1940. Localidade-tipo: Brasil, Mato Grosso do Sul, Salobra. Distribuição: Brasil (Mato Grosso do Sul). Ref.: Amorim \& Oliveira (2013). Oliveira \& AMORIM (2014) erroneamente incluíram na distribuição da espécie Neoempheria plaumanni Edwards os estados de São Paulo e Mato Grosso do Sul, no Brasil, e as províncias de Salta e Tucumán, na Argentina. A distribuição atual da espécie é aquela previamente mencionada em PAPAVERO (1978c).

\section{Leiinae \\ Leia Meigen, 1818}

fuscicornis Edwards, 1941. Localidade-tipo: Brasil, Mato Grosso do Sul, Salobra. Distribuição: Brasil (Mato Grosso do Sul). Ref.: AMORIM \& OliveIRA (2013).

salobrensis Edwards, 1941. Localidade-tipo: Brasil, Mato Grosso do Sul, Salobra. Distribuição: Brasil (Mato Grosso do Sul). Ref.: AMORIM \& OliveIRA (2013), comentários sobre a série-típica e etiquetas.

stonei Lane, 1958. Localidade-tipo: Bolívia, Mapiri, Sarampiuní, 700 m. incompleta Edwards, 1933 (preocc. Curran, 1928). Localidade-tipo: Bolívia, Mapiri, 
Sarampiuní, 700 m. Distribuição - Brasil (Mato Grosso do Sul), Bolívia (Mapiri).

\section{Tetragoneura Winnertz, 1846}

flavicauda Edwards, 1941. Localidade-tipo: Brasil, Mato Grosso do Sul, Salobra. Distribuição: Brasil (Mato Grosso do Sul), Equador (Napo). Ref.: Duret (1980); AMORIM \& Oliveira (2013).

\section{Mycetophilinae Epicypta Winnertz, 1863 Epicypta (Boscmyia) Lane, 1954}

forattinii Lane, 1960. Localidade-tipo: Brasil, Mato Grosso do Sul, Bataguassu, Rio Três Barras. Distribuição: Brasil (Mato Grosso do Sul).

\section{Epicypta (Callicypta) Lane, 1954}

anambesi Lane, 1960. Localidade-tipo: Brasil, Mato Grosso do Sul, Bataguassu, Rio Caraguatá. Distribuição: Brasil (Mato Grosso do Sul).

punctulata (Lane, 1947). Localidade-tipo: Brasil, Mato Grosso do Sul, Maracaju. Distribuição: Brasil (Mato Grosso do Sul).

\section{Epicypta (Epicypta) Winnertz, 1863}

planiventris (Enderlein, 1910). Localidade-tipo: Brasil, Santa Catarina. Distribuição: Brasil (Mato Grosso do Sul, Santa Catarina). Ref.: EDWARDS (1941).

\section{Neallodia Edwards, 1932}

brevicornis (Enderlein, 1910). Localidade-tipo: Brasil, Santa Catarina. Distribuição: Brasil (Mato Grosso do Sul, Santa Catarina). Ref.: EdwARds (1941); AMORIm \& OliveIrA, (2013).

\section{DISCUSSÃO}

A fauna de Bibionomorpha na Região Neotropical, como para a maior parte de Diptera, é conhecida principalmente das regiões Sul e Sudeste do Brasil. Isso é resultado da concentração de pesquisadores em taxonomia do grupo nos estados de São Paulo e Rio de Janeiro, além de coletas históricas em Santa Catarina e áreas do sul da Floresta Atlântica (mais recentemente, com o projeto FAPESP 2003/12.074-9). É necessário dar destaque também às coletas regulares e estudo da fauna de Diptera da Amazônia (Projeto PRONEX/AM, CNPq 016/2006).

Os registros de famílias de Bibionomorpha para o estado do Mato Grosso do Sul advém especialmente dos trabalhos dos entomólogos Frederick Wallace Edwards e John Lane, a partir de algum material coletado entre $50 \mathrm{e}$ 90 anos atrás. Há necessidade de avançar no conhecimento de fauna da região Centro-Oeste do Brasil em termos de áreas amostradas, bem como na intensidade de coleta para a construção de acervos representativos da diversidade do grupo no estado. Há uma perspectiva de mudança desse cenário com o projeto "Diptera dos estados do Mato Grosso, Mato Grosso do Sul e Rondônia: diversidade, sistemática e limites distribucionais", no âmbito de uma rede temática financiada pelo CNPq/FAPESP (SISBIOTA CNPq 563256/2010-9/ FAPESP 2010/52314-0).

Material com parte dessa fauna encontra-se depositada em museus e outras instituições no Brasil e no exterior. A coleção mais significativa de Diptera é a do Museu de Zoologia da Universidade de São Paulo (MZUSP), seguida pelas coleções de entomologia do Instituto Nacional de Pesquisas Amazônicas (INPA), Museu Paraense Emílio Goeldi (MPEG), Museu Nacional da Universidade Federal do Rio de Janeiro (QBUM), a coleção entomológica Padre Jesus Santiago Moure do Departamento de Zoologia da Universidade Federal do Paraná (DZUP). Em termos de material de referência (holótipos e parátipos), a coleção brasileira mais significativa é a do MZUSP. Algumas coleções fora do Brasil alocam uma quantidade significativa de material neotropical, como a coleção do Natural History Museum (NHM), Londres, Reino Unido; Muséum national d'Histoire Naturelle (MNHN), Paris, França; Canadian National Collection of Insects, Aracnids and Nematods (CNC), Ottawa, Canadá; Staatlischen Museum fur Naturkunde (SMNS), Stuttgart, Alemanha, e National Museum of Natural History (USNM), Washington DC, Estados Unidos da América.

Como visto acima, até 2014 a fauna de Bibionomorpha do Mato Grosso do Sul era conhecida apenas por representantes de Anisopodidae, Sciaridae, Keroplatidae e Mycetophilidae, não havendo quaisquer registros de Bibionidae, Cecidomyiidae, Rangomaramidae, Ditomyiidae, Diadocidiidae e Lygistorrhinidae. Mesmo no caso de Anisopodidae, Sciaridae, Keroplatidae e Mycetophilidae, a riqueza de espécies desses grupos conhecida até agora para a região Centro-Oeste do Brasil é apenas uma fração da diversidade real. De fato, a ausência de registros para essas famílias se repete nos outros estados da região Centro-Oeste, reflexo da ausência de coletas regulares, coleções organizadas e identificadas e taxonomistas, o que se aplica também às outras ordens de insetos no Brasil (CARVALHO, 1998).

No que concerne à fauna de Bibionomorpha, os catálogos indicam registros de espécies para os estados de Goiás, Mato Grosso e Distrito Federal que se espera que ocorram no Mato Grosso do Sul. Isso permite, assim, uma listagem ligeiramente mais ampla da diversidade do grupo no estado. Anisopodidae: Olbiogaster hallucinata Tozoni, Olbiogaster scalaris (Wiedemann), Sylvicola guttatus (Schiner). Bibionidae: Bibionellus barrettoi Lane \& Forattini, Enicoscollus hardyi Fitzgerald, Plecia brazilana Hardy, Plecia impilosa Hardy. Cecidomyiidae: Termitomastus leptoproctus Silvestri, Schizomyia tuiuiu Urso-Guimarães \& Amorim. Sciaridae: Bradysia bororoi (Lane), Bradysia janduisi (Lane), Trichomegalosphys ribeiroi (Lane). Rangomaramidae: Colonomyia sp. Ditomyiidae: Australosymmerus (Melosymmerus) guarani (Lane), Australosymmerus (Melosymmerus) tupi (Lane). 
Keroplatidae: Heteropterna abdominalis Lane, Heteropterna trileuca Edwards, Isoneuromyia argenteotomentosa (Kertész), Isoneuromyia goianensis (Lane), Isoneuromyia xanthocera (Edwards), Lyprauta amazonensis (Lane), Macrocera guarani Lane, Macrocera plaumanni Edwards, Neoceroplatus hodeberti Matile, Placoceratias barrettoi (Lane), Plautyra macilenta (Lynch Arribálzaga), Proceroplatus catharinae (Edwards), Proceroplatus terenoi (Lane). Taulyrpa vespiformis (Enderlein), Xenoplatyura coheri (Lane). Lygistorrhinidae: Lygistorrhina (Probolaues) barrettoi Lane. Mycetophilidae: Mycomya americana (Schiner), Mycomya brontes Coher, Mycomya hamadryas Coher, Neoempheria depleta Coher, Neoempheria goiana Coher, Neoempheria social Coher, Leia aruaci Lane, Leia biamputata Edwards, Leia bilunula Wiedemann, Leia cayapoi Lane, Leia diversicornis (Kertész), Leia halterata (Kertész), Leia picticornis (Kertész), Leiella unicincta Edwards, Manota palpalis Lane, Exechia goianensisi (Lane), Epicypta (Epicypta) aczeli Lane, Epicypta (Epicypta) brasiliana (Edwards), Epicypta (Epicypta) goianensis (Lane), Sceptonia longicornis Enderlein, Schnusea sessilis Edwards, Schnusea desanei Lane.

Embora a riqueza da fauna e flora do Brasil seja propalada com frequência, o conhecimento sobre essa biodiversidade é proporcionalmente muito pequeno (Lewinsohn \& Prado, 2005). As espécies estão sendo extintas a uma velocidade muito maior do que conseguimos descobri-las e descrevê-las (CARVALHO et al., 2008), sendo que a insuficiência de taxonomistas para executar esse trabalho apenas agudiza essa situação (WILSON, 1987; PlatNICK, 1992). Infelizmente, a pressão antrópica sobre as áreas naturais é cada vez mais acelerada, com impactos ambientais diretos causando distúrbios nos diversos hábitats, com resultados devastadores mesmo num país megadiverso como o Brasil (CARVAlHo, 2011). A fauna de Diptera é apenas um exemplo isolado de um processo mais amplo. A pressão sobre as áreas com floresta semidecídua, como é o caso do Mato Grosso do Sul, é ainda mais grave, tornando-as uma das áreas mais ameaçadas do mundo (AMORIM et al., 2010).

Agradecimentos. Os autores agradecem à Fundação de Apoio ao Desenvolvimento do Ensino, Ciências e Tecnologia do Estado de Mato Grosso do Sul (Fundect) e a Superintendência de Ciências e Tecnologia do Estado de Mato Grosso do Sul (Sucitec/MS) pelo convite de participação neste fascículo especial da Iheringia, Série Zoologia e o suporte financeiro para sua publicação. Este trabalho recebeu apoio financeiro do Conselho Nacional de Desenvolvimento Científico e Tecnológico (CNPq, processo SISBIOTA 563256/2010-9) e da Fundação de Amparo à Pesquisa do Estado de São Paulo (FAPESP), processos: SSO (2008/52342-6 e 2012/515773), RLF (2007/59466-8, 2013/16524-9 e CNPq 159016/2012-6), MVUG (99/01429-1), DSA (2003/12.074-9), SISBIOTA (2010/52314-0).

\section{REFERÊNCIAS BIBLIOGRÁFICAS}

Amorim, D. S. 1992. A catalogue of the family Sciaridae (Diptera) in the Americas South of the United States. Revista Brasileira de Entomologia 36(1):5-77.

Amorim, D. S. 1993. A phylogenetic analysis of the basal groups of Bibionomorpha, with a critical reanalysis of the wing vein homology. Revista Brasileira de Biologia 52(3):379-399.
Amorim, D. S. 1994. A new suprageneric classification of the Scatopsidae (Diptera: Psychodomorpha). Iheringia, Série Zoologia (77):107-112.

Amorim, D. S. \& Falaschi, R. L. 2012. Catalogue of Neotropical Diptera. Rangomaramidae. Neotropical Diptera 21:1-7.

Amorim, D. S. \& Oliveira, S. S. 2008. Eleven new species of the genus Cluzobra Edwards (Diptera, Mycetophilidae, Sciophilinae) from the Atlantic Forest of Brazil. Zootaxa 1920:1-28.

Amorim, D. S. \& Oliveira, S. S. 2013. Types of Neotropical Mycetophilidae (Diptera) at the Natural History Museum collection, London. Zootaxa 3726:1-119.

Amorim, D. S.; Oliveira, S. S. \& Balbi, M. I. P. A. 2008a. Azana atlantica, n.sp., with reduced mouthparts and two ocelli: first record of Azana for the Neotropical region (Diptera: Mycetophilidae: Sciophilinae). Zootaxa 1789:57-65.

Amorim, D. S.; Oliveira, S. S. \& Balbi, M. I. P. A. 2008b. First Neotropical species of genus Azana (Diptera: Mycetophilidae: Sciophilinae). Zootaxa 1937:67-68.

Amorim, D. S. \& Rindal, E. 2007. A phylogenetic study of the Mycetophiliformia, with creation of the subfamilies Heterotrichinae, Ohakuneinae, and Chiletrichinae for the Rangomaramidae (Diptera, Bibionomorpha). Zootaxa 1535:1-92.

Amorim, D. S.; Silva, V. C. \& Balbi, M. I. P. A. 2002. Estado do conhecimento dos Diptera neotropicais. In: Costa, C.; VAnIN, S. A.; Lobo, J. M. \& Melic, A. eds. Proyecto de Red Iberoamericana de Biogeografia y Entomología Sistemática, PRIBES 2002. Zaragoza, Sociedad Entomológica Aragoneza, CYTED, p.29-36.

Amorim, D. S. \& Tozoni, S. H. S. 1994. Phylogenetic and biogeographic analysis of the Anisopodoidea (Diptera, Bibionomorpha), with an area cladogram for intercontinental relationships. Revista Brasileira de Entomologia 38:517-543.

Amorim, D. S. \& YeATES, D. 2006. Pesky gnats: ridding dipteran classification of the "Nematocera". Studia Dipterologica 13(1/2):3-9.

Amorim, D. S.; Papavero, N.; Silva, V. C.; Lamas, C. J. E.; Nihei, S. S.; Ribeiro, G. C.; Santos, C. M. D.; Fonseca, H. M.; Coscarón, S.; Balbi, M. I. P. A.; Falashi, R. L.; Oliveira, S. S.; Capellari, R. S.; Ament, D. C.; Riccardi, P. R.; Conti, C. F.; Mellinger, G. B.; Mello, R. J. C. L.; Pinto, Â. P.; Rodrigues, P. F. M.; Cezar, L. A.; Yamaguchi, C.; Garcez, R. N.; Morimoto, F. T. S.; Figueiredo, R. R.; Araya, N. C. C.; Arruda, P. M.; Nunes, R. F. \& Andrade, M. R. 2010. A large scale survey of the Diptera of the Atlantic Forest. In: VII International Congress of Dipterology. San José, Costa Rica, p. 9-13.

Bechev, D. \& Chandler, P. J. 2011. Catalogue of the Bolitophilidae and Diadocidiidae of the World (Insecta: Diptera). Zootaxa 2741:38-58.

Bertone, M. A.; Courtney, G. W. \& Wiegmann, B. M. 2008. Phylogenetics and temporal diversification of the earliest true flies (Insecta: Diptera) based on multiple nuclear genes. Systematic Entomology 33(4):668687.

Brown, B. V. 2005. Malaise Trap Catches and the Crisis in Neotropical Dipterology. American Entomologist 51(3):180-183.

Brown, B. V.; Borkent, A.; Cumming, J. M.; Wood, D. M.; Woodley, N. E. \& Zumbado, M. 2009. Manual of Central American Diptera. Ottawa, NRC Research Press. Vol. 1, 714p.

Carvalho, C. J. B. 1998. Taxonomista de insetos, uma espécie em extinção no Brasil. Informativo da Sociedade Entomológica do Brasil 23(1):14.

Carvalho, C. J. B. 2011. Padrões de endemismo e a conservação da biodiversidade. Megadiversidade 5(1/2):77-86

Carvalho, M. R.; Bockmann, F. A.; Amorim, D. S. \& Brandão, C. R. F. 2008. Systematics must embrace comparative biology and evolution, not speed and automation. Journal Evolutionary Biology 35:97-104.

Chandler, P. J. 2002. Heterotricha Loew and allied genera (Diptera: Sciaroidea): offshoots of the stem group of Mycetophilidae and/or Sciaridae? Annales de la Societé Entomologique de France 38:101144.

Coher, E. I. 2011. A New Genus and species of North American Robsonomyiini (Diptera: Sciaroidea: Keroplatidae: Macrocerinae) from the Florida Keys. Insecta Mundi 198:1-6.

Curran, C. H. 1928. Insects of Porto Rico and the Virgin Islands. Diptera or two-winged flies. In: New York Academy of Sciences, Scientific Survey of Puerto Rico and The Virgin Islands, 11. New York. part 1. p.1-118. 
Duret, J. P. 1980. Notas sobre el género Tetragoneura Winnertz, 1846 (Diptera, Mycetophilidae). Acta Zoologica Lilloana 36:103-111.

EDWARDS, F.W. 1941. Mycetophilidae (Diptera) collected by the expedition to Matto Grosso of Brazilian Zoological Club, in July 1939. Revista de Entomologia 12(1-2):303-314.

Evenhuis, N. L. 1994. Catalogue of the fossil flies of the world (Insecta: Diptera). Leiden, Backhuys Press, 600p.

Evenhuis, N. L. 2006. Catalog of the Keroplatidae of the World (Insecta: Diptera). Bishop Museum Bulletin in Entomology 13:1-178.

FALASCHI, R. L. 2008. Revisão e análise filogenética de Melosymmerus Munroe (Diptera, Bibionomorpha, Ditomyiidae). Dissertação de Mestrado. Ribeirão Preto, Universidade de São Paulo, 89p.

FALASCHI, R. L. 2012. Relações filogenéticas entre os Keroplatinae: posição de Orfeliini e relação entre seus gêneros (Diptera: Keroplatidae). Tese de Doutorado. Ribeirão Preto, Universidade de São Paulo, 213p

FALASCHI, R. L. \& Amorim, D. S. 2009. Review of the Neotropical species of Nervijuncta Marshall, 1896 (Diptera, Ditomyiidae). Zootaxa 2219:1830 .

FitzGerald, S. J. 1997. A new species of Enicoscolus (Diptera: Bibionidae) from Brazil, with additional distribution records for the genus. PanPacific Entomologist 73(3):152-155.

Fitzgerald, S. J. 2004. Evolution and Classification of Bibionidae (Diptera: Bibionomorpha). Ph. D. dissertation. Corvallis, Oregon State University, 385p.

FitzGerald, S. J. 2009. Bibionidae (March flies, Love-bugs). In: Brown, B. V.; Borkent, A.; Cumming, J. M.; Wood, D. M.; Woodley N. E. \& Zumbado, M. A. eds. Manual of Central American Diptera. Ottawa, NRC Research Press. p.245-251. vol. 1

Gagné, R.J. \& M. Jaschrof. 2014. A Catalog of the Cecidomyiidae (Diptera) of the World. 3rd Edition. Digital version 2.

Hancock, E. G. \& Amorim, D. S. 2009. Anisopodidae (Wood gnats or Window gnats). In: Brown, B. V.; Borkent, A.; Cumming, J. M.; Wood, D. M.; Woodley N. E. \& Zumbado, M. A. eds. Manual of Central American Diptera. Ottawa, NRC Research Press. Vol. 1, p.341-345

Hardy, D. E. 1966. Family Bibionidae. In: Papavero, N. ed. A Catalogue of the Diptera of the Americas south of the United States. São Paulo, Departamento de Zoologia da Secretaria da Agricultura do Estado de São Paulo, p.1-20.

Hennig, W. 1973. Diptera (Zweiflügler). Handbuch der Zoologie 4(2)2/31:1-337.

HipPa, H. \& VilkamaA, P. 2005. The genus Sciarotricha gen. n. (Sciaridae) and the phylogeny of recent and fossil Sciaroidea (Diptera). Insect Systematics and Evolution 36:121-144.

Hippa, H.; Mattsson, I. \& VilkamaA, P. 2005. New taxa of the Lygistorrhinidae (Diptera: Sciaroidea) and their implications for a phylogenetic analysis of the family. Zootaxa 960:1-34.

Huerta, H. \& Ibañez-Bernal, S. 2008. The family Lygistorrhinidae (Diptera: Sciaroidea) in Mexico and the description of two new species. Zootaxa 1808:44-52.

JASCHHOF, M. \& Didham, R. K. 2002. Rangomaramidae fam. nov. from New Zealand and implications for the phylogeny of the Sciaroidea (Diptera: Bibionomorpha). Studia Dipterologica (Supplement) 11:1-60.

JASCHHOF, M. \& JASCHHOF, C. 2007. On the genus Diadocidia (Diptera, Sciaroidea, Diadocidiidae) in Costa Rica. Zootaxa 1586:33-38.

LANE, J. 1948. Ceroplatinae da Região Neotropical. (Diptera, Mycetophilidae). Revista Brasileira de Entomologia 19(3):437-458

Lewinsohn, T. \& Prado, P. I. 2005. Quantas espécies há no Brasil? Megadiversidade 1:36-42.

Matile, L. 1971. Une nouvelle tribu de Mycetophilidae: les Metanepsiini (Dipt.). Bulletin de la Société Entomologique de France 76:91-97.

Matile, L. 1981. Description d'un Keroplatidae du crétacé moyen et données morphologiques et taxinomiques sur les Mycetophiloidea (Diptera). Annales de la Société Entomologique de France 17:99-123.

Matile, L. 1989. Superfamily Sciaroidea. In: Evenhuis, N. L. \& BRILL, E. J. eds. Catalog of the Diptera of the Australasian and Oceanic Regions. Honolulu, Bishop Museum Press, p.123-145.

Matile, L. 1990. Recherches sur la systématique et l'évolution des Keroplatidae (Diptera, Mycetophiloidea). Memóires du Muséum National d'Histoire Naturelle, Serie A, Zoologie 148:1-682.
Menzel, F.; Smith, J. E. \& Colauto, N. B. 2003. Bradysia difformis Frey and Bradysia ocellaris (Comstock): Two additional neotropical species of black fungus gnats (Diptera: Sciaridae) of economic importance: A redescription and review. Annals of the Entomological Society of America 96(4):448-457.

Mohrig, W. \& Menzel, F. 2009. Sciaridae. In: Brown, B.V.; Borkent, A.; Cumming, J. M.; Wood, D. M.; Woodley N. E. \& Zumbado, M. A. eds. Manual of Central American Diptera. Ottawa, NRC Research Press. Vol. 1, p.279-293.

Munroe, D. D. 1974. The systematics, phylogeny, and zoogeography of Symmerus Walker and Australosymmerus Freeman (Diptera: Mycetophilidae: Ditomyiidae). Memoirs of Entomological Society of Canada 92:1-183

Oliveira, S. S. 2009. Dziedzickia Johannsen e Schnusea Edwards (Diptera, Mycetophilidae): diversidade das espécies neotropicais e análise filogenética. Dissertação de Mestrado. Ribeirão Preto, Universidade de São Paulo, 145p.

Oliveira, S. S. \& Amorim, D. S. 2010a. The genus Phthinia Winnertz (Diptera, Mycetophilidae) in the Neotropical region, with the first records from Brazil. Zootaxa 2350:22-34.

Oliveira, S. S. \& Amorim, D. S. 2010b. Four new species of Paratrizygia Tonnoir from the Brazilian Atlantic Forest (Diptera, Mycetophilidae, Sciophilinae). Zootaxa 2629:29-34.

Oliveira, S. S. \& Amorim, D. S. 2011. Docosia adusta sp. nov. (Diptera: Mycetophilidae) from the Colombian Andes: a Holarctic element in northwestern South America. The Canadian Entomologist 143:688696.

Oliveira, S. S. \& Amorim, D. S. 2012a. Catalogue of Neotropical Lygistorrhinidae. Neotropical Diptera 22:1-4.

Oliveira, S. S. \& Amorim, D. S. 2012b. Six new species of Paraleia Tonnoir (Diptera, Mycetophilidae): amphinotic elements at the northern range of the Andes. Zootaxa 3186:1-24.

Oliveira, S. S. \& Amorim, D. S. 2014. Catalogue of Neotropical Diptera. Mycetophilidae. Neotropical Diptera 25:1-87.

Oosterbroek, P. \& Courtney, G. 1995. Phylogeny of the nematocerous families of Diptera (Insecta). Zoological Journal of the Linnean Society 115:267-331

OzDIKMEN, H. \& BASAR, K. 2011. A new name for the Neotropical genus Aniarella Enderlein (Diptera, Sciaridae). Revista Brasileira de Entomologia 55(1):15-16.

Papavero, N. 1967. Family Anisopodidae. In: Papavero, N. ed. A Catalogue of the Diptera of the Americas south of the United States. São Paulo, Departamento de Zoologia da Secretaria da Agricultura do Estado de São Paulo, p.1-9.

Papavero, N. 1977a. Family Diadocidiidae. In: Papavero, N. ed. A Catalogue of the Diptera of the Americas south of the United States. São Paulo, Departamento de Zoologia da Secretaria da Agricultura do Estado de São Paulo, p.1-3.

Papavero, N. 1977b. 19D. Family Lygistorrhinidae. In: PapaVero, N. ed. A Catalogue of the Diptera of the Americas south of the United States. São Paulo, Departamento de Zoologia da Secretaria da Agricultura do Estado de São Paulo, p.1-2.

Papavero, N. 1978a. Family Ditomyiidae. In: Papavero, N. ed. A Catalogue of the Diptera of the Americas south of the United States. São Paulo, Departamento de Zoologia da Secretaria da Agricultura do Estado de São Paulo, p.1-6.

PapaVero, N. 1978b. 19C. Family Keroplatidae (Ceroplatidae, incl Macroceridae). In: PAPAVEro, N. ed. A Catalogue of the Diptera of the Americas south of the United States. São Paulo Museu de Zoologia, Universidade de São Paulo, p.1-22.

Papavero, N. 1978c. 19E. Family Mycetophilidae. In: Papavero, N. ed. A Catalogue of the Diptera of the Americas south of the United States. São Paulo, Museu de Zoologia, Universidade de São Paulo, p.1-78.

PAPAVERO, N. 2002. Insecta, Diptera, Lygistorrhinidae. Fauna da Amazônia Brasileira 10:1.

Pape, T.; Blagoderov, V. \& Mostovski, M. B. 2011. Order Diptera Linnaeus, 1758. In: Animal biodiversity: An outline of higher-level classification and survey of taxonomic richness (Z.-Q. Zhang, ed.). Zootaxa 3148:222-229.

PAPP, L. 2008. Micromacrocera gen. n., the smallest macrocerine fly (Diptera: Keroplatidae, Macrocerinae). Acta Zoologica Academiae Scientiarum Hungaricae 54(1):13-21. 
PAPP, L. 2010. A study on Hesperinus Walker with description of a new species (Diptera: Hesperinidae). Acta Zoologica Academiae Scientiarum Hungaricae 56(4):347-370.

PAPP, L. \& ŠEvČíK, J. 2005. Sciarokeroplatinae, a new subfamily of Keroplatidae (Diptera). Acta Zoologica Academiae Scientiarum Hungaricae 51(2):113-123.

Pinto, L. G. \& Amorim, D. S. 2000. Bibionidae (Diptera: Bibionomorpha). Morfologia e análise filogenética. Ribeirão Preto, Holos. 98p.

Platnick, N. I. 1992. Patterns of Biodiversity. In: Eldredge, N. ed. Systematic, Ecology, and the Biodiversity Crisis. New York, Columbia University Press, p. 15-24.

Rindal, E.; Søli, G. E. \& BachmanN, L. 2009. Molecular phylogeny of the fungus gnat family Mycetophilidae (Diptera, Mycetophiliformia). Systematic Entomology 34:524-532.

ŠEVČ́́K, J. 2009. Langkawiana maculata gen. et sp. n. from Malaysia and its systematic position in the tribe Robsonomyiini (Diptera: Keroplatidae). Zootaxa 2221:58-66.

ŠEvČíK,, J. \& PAPP, L. 2009. Microkeroplatus, a new genus of Keroplatidae (Diptera) from the oriental region. Acta Zoologica Academiae Scientiarum Hungaricae 55(4):339-347.

ŠEvČík, J. \& HIPPA, H. 2010. New species of Chalastonepsia and Pectinepsia gen. nov. (Diptera: Mycetophilidae) from the Oriental and Australasian Regions. Acta Entomologica Musei Nationalis Pragae 50(2):595-608.

SøLI, G. E. E. 1997. The adult morphology of Mycetophilidae (s. str.), with a tentative phylogeny of the family (Diptera, Sciaroidea). Entomologica Scandinavica (Suppl.) 50:5-55.

Tozoni, S. H. S. 1993. Revision of the genus Olbiogaster Osten-Sacken (Diptera, Anisopodoidea, Olbiogastridae). I. Description of 13 new species, taxonomical notes, and a key to the Neotropical species. Revista Nordestina de Biologia 8(2):119-142.

Tozoni, S. H. S. 1998. Sistemática filogenética dos Mycetophilidae
(Diptera: Bibionomorpha). Tese de Doutorado. Curitiba, Universidade Federal do Paraná, 124p.

Tuomikoski, R. 1961. Zur Systematik der Bibionomorpha (Dipt.) I. Anisopodidae und Protorhypidae. Annales Entomologicae Fennicae 27:65-69.

Tuomikoski, R. 1966. Generic taxonomy of the Exechiini (Dipt., Mycetophillidae). Annales Entomologicae Fennicae 32:159-194.

VÄISÄNEN, R. 1984. A monograph of the genus Mycomyia Rondani in the Holarctic region (Diptera, Mycetophilidae). Acta Zoologica Fennica 177:1-346.

VilkamaA, P. \& HipPA, H. 2004. The genus Xenosciara gen. n. and the phylogeny of the Sciaridae (Diptera). Zootaxa 699:1-24.

Wiegmann, B. M.; Trautwein, M. D.; Winkler, I. S.; Barr, N. B.; Kim, J. W.; Lambkin, C.; Bertone, M. A.; Cassel, B. K.; Bayless, K. M.; Heimberg, A. M.; Wheeler, B. M.; Peterson, K. J.; Pape, T.; Sinclair, B. J.; Skevington, J. H.; Blagoderov, V.; Caravas, J.; Kutty, S. N.; Schmidt- Otт , U.; Kampmeiern, G. E.; Thompson, C.; Grimaldi, D. A.; Beckenbach, A.T.; Courtney, G. W.; Friedrich, M.; Meier, R. \& Yeates, D. K. 2011. Episodic radiations in the fly tree of life. Proceedings of the National Academy of Sciences of the United States of America 108(14):5690-5695.

Wilson, E. O. 1987. Time to revive systematics. Science 230:1227.

Wood, M. \& Borkent, A. 1989. Phylogeny and classification of the Nematocera. In: McAlpine, J. F.; Peterson, B. V.; Shewell, G. E.; Teskey, H. J.; Vockeroth, J. R. \& Wood, D. M. eds. Manual of Neartic Diptera. Ottawa, Research Branch. Agriculture Canada, p. $1333-1370$.

Woodley, N. E. 1989. Phylogeny and classification of "orthorrhaphous" Brachycera. In: McAlpine, J. F.; Peterson, B. V.; Shewell, G. E.; Teskey, H. J.; Vockeroth J. R. \& Wood, D. M. eds. Manual of Nearctic Diptera. Ottawa, Research Branch, Agriculture Canada, p.1371-1395. 